\title{
Combined In Silico and In Vivo Analyses Reveal Role of Hes1 in Taste Cell Differentiation
}

\author{
Masato S. Ota ${ }^{1,2}$, Yoshiyuki Kaneko ${ }^{3}$, Kaori Kondo ${ }^{1,2}$, Soichi Ogishima ${ }^{3}$, Hiroshi Tanaka ${ }^{3}$, Kazuhiro Eto ${ }^{1}$, \\ Takashi Kondo ${ }^{2 *}$
}

1 Section of Molecular Craniofacial Embryology, Tokyo Medical and Dental University, Bunkyo-ku, Tokyo, Japan, 2 Kondo Research Unit, Neuro-Developmental Disorder Research Group, Brain Science Institute, Institute of Physical and Chemical Research (RIKEN), Wako, Saitama, Japan, 3 Department of Bioinformatics, Tokyo Medical and Dental University, Bunkyo-ku, Tokyo, Japan

\begin{abstract}
The sense of taste is of critical importance to animal survival. Although studies of taste signal transduction mechanisms have provided detailed information regarding taste receptor calcium signaling molecules (TRCSMs, required for sweet/ bitter/umami taste signal transduction), the ontogeny of taste cells is still largely unknown. We used a novel approach to investigate the molecular regulation of taste system development in mice by combining in silico and in vivo analyses. After discovering that TRCSMs colocalized within developing circumvallate papillae (CVP), we used computational analysis of the upstream regulatory regions of TRCSMs to investigate the possibility of a common regulatory network for TRCSM transcription. Based on this analysis, we identified Hes 1 as a likely common regulatory factor, and examined its function in vivo. Expression profile analyses revealed that decreased expression of nuclear HES1 correlated with expression of type II taste cell markers. After stage E18, the CVP of Hes $1^{-1-}$ mutants displayed over 5 -fold more TRCSM-immunoreactive cells than did the CVP of their wild-type littermates. Thus, according to our composite analyses, Hes 1 is likely to play a role in orchestrating taste cell differentiation in developing taste buds.
\end{abstract}

Citation: Ota MS, Kaneko Y, Kondo K, Ogishima S, Tanaka H, et al. (2009) Combined In Silico and In Vivo Analyses Reveal Role of Hes1 in Taste Cell Differentiation. PLoS Genet 5(4): e1000443. doi:10.1371/journal.pgen.1000443

Editor: Thomas E. Finger, University of Colorado Denver, United States of America

Received September 25, 2008; Accepted March 2, 2009; Published April 3, 2009

Copyright: () 2009 Ota et al. This is an open-access article distributed under the terms of the Creative Commons Attribution License, which permits unrestricted use, distribution, and reproduction in any medium, provided the original author and source are credited.

Funding: This work was supported by a grant from "The lijima Memorial Foundation for the Promotion of Food Science and Technology" and "The Society for Research on Umami Taste" to TK. The funders had no role in study design, data collection and analysis, decision to publish, or preparation of the manuscript.

Competing Interests: The authors have declared that no competing interests exist.

*E-mail: TKondo@brain.riken.jp

\section{Introduction}

Taste is one of the major chemosensory systems enabling animals to perceive crucial environmental stimuli. It performs the vital role of helping animals to identify favorable nutrition sources, as well as to avoid toxic substances, making taste a fundamental sensory recognition system that is required for survival $[1,2]$. While the ontogeny of the other special sense organs has been studied in depth at a molecular level [3-5], the development of taste remains to be clarified.

Taste buds are the sensory end organs for gustation, and are located on the epithelium of the tongue and palate. On the tongue, they reside on three types of papillae, i.e., fungiform, foliate, and circumvallate $[2,6,7]$. In adult mammals, each taste bud comprises groups of 50-100 spindle-shaped epithelial cells and a small number of proliferative cells $[8,9]$. Taste bud cells are heterogeneous in terms of gene expression profiling of individual taste cells, as well as in their ultrastructural characteristics. [8,10-14]. Ultrastructual studies have revealed three distinct anatomical types of spindle-shaped epithelial cells within each taste bud: type I (dark), type II (light), and type III (intermediate) cells [8,10,11]. Type II cells have a characteristic large round nucleus and are responsible for the sweet, bitter, and umami taste sensations [2,68,10]. These cells express a number of $G$ protein-coupled receptors and common downstream transduction components called taste receptor calcium signaling molecules (TRCSMs; e.g.,
PLC $\beta 2$, gustducin [GNAT3], and IP3R3 [ITPR3]) [2,6,7,10]. Although several studies have examined the lineage of taste cells $[8,10,15,16]$, the molecular mechanisms of cell differentiation in developing taste buds have remained elusive. We took a novel approach toward investigating taste cell development in mice by combining in silico and in vivo analyses of the TRCSM transcription regulatory network in type II taste cells.

\section{Results}

\section{Early Phase of TRCSM Expression}

We examined the expression of TRCSMs in the epithelium of presumptive circumvallate papillae (CVP) during mouse embryogenesis. The papilla structure of CVP is already visible before embryonic day 14 (E14) [17]. We examined expression of five TRCSMs - PLCß2, gustducin, IP3R3, Ggammal3 (GNG13), and Trpm5 - in developing CVP by immunohistochemistry and/or in situ hybridization. We identified the appearance of cells expressing these TRCSMs (which are widely accepted as representative markers for differentiated taste cells) in serial sections from the posterior one-third of the embryonic tongue (Figure 1) $[2,6,7,10]$. One series of sections from an entire CVP was subjected to each combination of antibodies or probes, such as PLC $\beta 2$ and IP3R3 antibodies, and more than five CVPs were subjected to histological analysis with each combination of markers at each developmental stage. 


\section{Author Summary}

The sensation of taste is composed of five basic modalities: sweet, bitter, umami, sour, and salty. Specialized taste cells perceive the various chemical cues within food. About 100 taste cells assemble into onion-shaped clusters called taste buds, which are located on taste papillae in the tongue epithelium and on oral mucosa. Of the five taste modalities, the taste stimulants responsible for sweet, bitter, and umami tastes are recognized by a group of $G$ protein-coupled taste receptors, and the signal transduction pathways utilized following receptor stimulation share common molecules. However, it is still largely unknown how these molecules are regulated during taste cell development. We performed computer analyses based on previously known information about signal transduction pathways involved in the taste-sensing system to identify taste stem cells/progenitor factors of type II taste cells (responsible for sweet, bitter, and umami taste sensations). We found several transcription factors likely to bind to the regulatory regions of taste-related calcium signaling molecules (TRCSMs), and identified Hes 1 as a potential candidate for common regulatory factors of TRCSMs. In vivo analyses using wild-type and Hes 1 mutant mice confirmed that Hes 1 regulates differentiation of bitter-, sweet-, and umami-sensing cells.

In previous studies, cytokeratin-8/Tromal (CK8) staining revealed that the taste bud primordia in CVP appear from E15 onward during early development of the tongue epithelium in mice [18]; this is before the morphology of taste buds becomes evident, approximately $2 \mathrm{~d}$ postnatal (P2). Although a search was carried out for TRCSMs within the mouse tongue epithelium from E11 to E16, none were detected; TRCSM-positive cells appear as single isolated cells among the cell population immunoreactive against GK8 at E17 (Figure S1 and Table S1). These results indicate that TRCSM-positive cells appear in the developing GVP just before birth in mice (Figure 1, S1 and S2). Around the time of birth (E18 to P0), two or three TRCSMpositive cells were observable within the entire CVP (Figures 1, 4 and S2). These cells were not considered to be fully differentiated taste cells because they lacked certain crucial taste cell markers such as taste cell receptors. While previous studies reported incomplete overlapping of five TRCSMs in taste buds in the CVP of adult mice [19-21], we detected $100 \%$ colocalization of these TRCSMs (PLCß2, gustducin, IP3R3, Ggamma13, and Trpm5) in the developing tongue epithelium, from E17 to at least P5 (Figures 1, S2 and Table S1). These results suggest that TRCSMs are expressed simultaneously in the same cell population during early development of the taste cell lineage in CVP.

\section{Computational Analysis of the Promoter Regions of TRCSMs}

The synchronous cellular colocalization of TRCSMs led us to investigate the regulatory mechanisms of TRCSMs, under the hypothesis that these genes are involved in the same regulatory network and share common regulatory factors, at least in the early phase of taste cell development. We analyzed the promoters of the five TRCSMs in silico to identify any common transcription factors that bind to regulatory sequences of taste stimuli signaling components. A series of putative transcription factor binding sites to these DNA sequences were identified by the Match program [22], which searched for regulatory sequences up to $5 \mathrm{~kb}$ upstream of each of the five TRCSMs (Figure 2). We further sieved common transcription factors through interspecies comparisons based on information acquired from mouse, rat, and human DNA sequences. Using these computational predictions, we identified 94 transcription factors as putative common transcription regulators (Figure 2A). These factors, which included candidates for factors implicated in the taste developmental system, are listed in Table S2. To evaluate this approach, we further performed a bibliographic and database search for gene expression within the embryonic oral epithelium. Because transcription repressors are presumably required to suppress the expression of TRCSMs in stem or precursor cells, we focused on transcription repressors within our list of identified candidates, in an effort to identify the regulator for taste stem cells or precursor cells. Ultimately, Hes1, a basic helix-loop-helix type of transcription factor, emerged as the most likely candidate from our different sets of informatics screenings (Figure S3).

\section{HES1 Binds the Promoters of $P I c \beta 2$ and $1 p 3 r 3$}

To confirm that HES1 binds to the $P l c \beta 2$ and $I p 3 r 3$ promoter regions (Figure 2B), we ran chromatin immunoprecipitation assays (ChIP) using an antibody against HES 1. We designed several pairs of primers to amplify putative HES1 binding sites in these promoter regions, as predicted by our in silico analyses. As controls, we also designed pairs of primers that did not contain the HES1 binding sequence (Figure 3). ChIP with the $\mathrm{pl}$ primer pair yielded a higher recovery of chromatin than did ChIP with the control p2C primer pair (Figure 3). Similarly, the ipl and ip2 primer pairs also yielded a higher recovery of chromatin than did the control ip3C primer pair. The ip3C control primers showed a relatively high recovery of chromatin, most likely due to the close location to the third HES 1 binding site, and to their position between two HES1 binding sites within the Ip3r3 promoter region (Figure 3). These results suggest that HES 1 bound the predicted sequences in the Plc $\beta 2$ and Ip $3 r 3$ promoter regions.

\section{Expression Analysis of Hes 1 in CVP}

Because our composite approach to identifying factors in the regulatory network of taste system development picked up Hes 1 as a strong candidate, we further analyzed the role of Hes 1 in taste system development. In situ hybridization analyses against tongue epithelium from 3-weeks-old animals (W3) revealed that large numbers of cells within taste buds exhibited Hes 1 transcript (Figure S4) [23], and that expression of Hes1 overlapped with the TRCSMs (data not shown). This observation contradicts somewhat the hypothesis that HES1 directly represses the expression of TRCSMs in taste buds; therefore, we performed detailed immunohistochemical analyses using HES1 antibody on GVPs from P0 animals and W3 animals (Figure 4). The TRCSMpositive cells observed at $\mathrm{P} 0$ showed a reduction in HES1 immunoreactivity within nucleus, suggesting that HESl protein had evacuated from nuclei (Figure 4). In W3 animals, HES1 localized in the cytoplasm of most taste bud cells (Figure 4). This cytoplasmic HES1 can be considered to be nonfunctional as a transcription regulator. The few cells that showed HES1 localized in the nucleus as well as in the cytoplasm exhibited no IP3R3 expression (Figure 4; indicated by white arrows), while the cells with cytoplasmic HES1 only also expressed IP3R3 (Figure 4; indicated by arrowheads). This suggested that regulation of the subcellular localization of HES1 was important for taste cell differentiation.

Because HES1 represses transcription from bound promoters, cells positive for HES 1 within the nucleus may be either precursor cells (including stem cells) of TRCSM-positive cells (type II; responsible for sweet, bitter, and umami taste) or other cell types within the taste cell lineage, such as type I or type III cells [10,11]. 

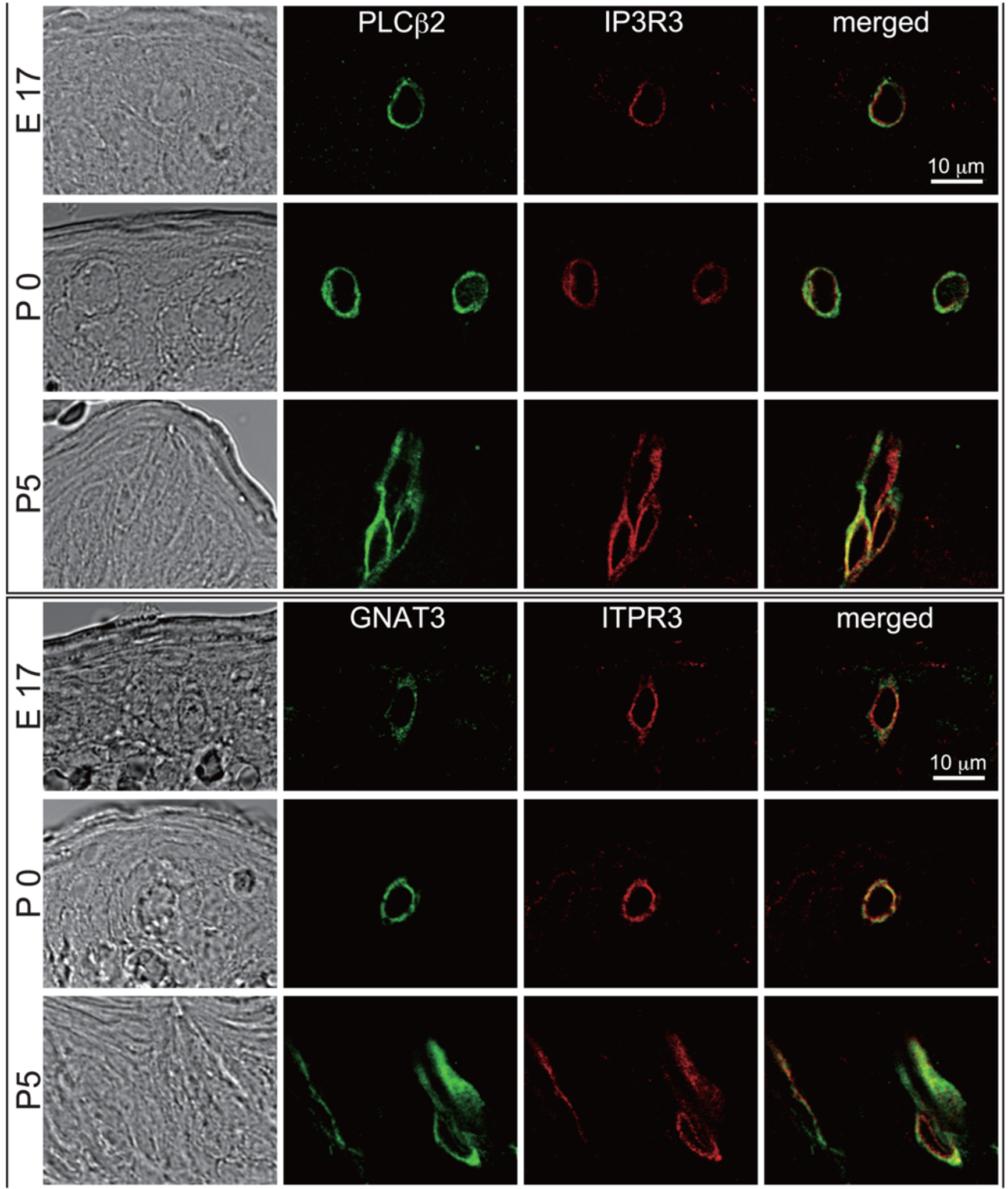

Figure 1. Colocalization of TRCSMs in early developing CVP. Immunohistochemical analyses of PLC $\beta 2$ (green signal) and IP3R3 or gustducin (red signal) in developing taste buds in CVP from stages E17 to P5. PLC 32 and IP3R3 or gustducin colocalize within the same cells, at least until P5. Scale bar, $10 \mu \mathrm{m}$.

doi:10.1371/journal.pgen.1000443.g001 

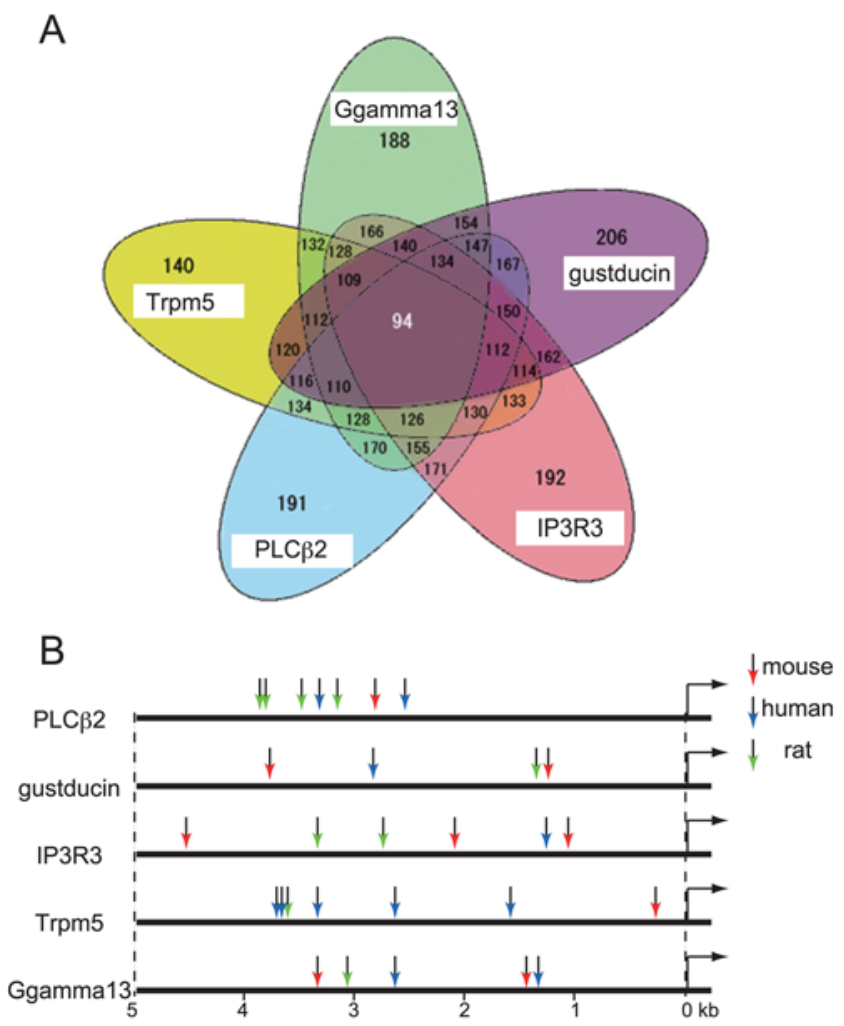

Figure 2. In silico analysis of the upstream region of TRCSMs in mammals. (A) Venn diagram representing the results of in silico analysis of the $5 \mathrm{~kb}$ upstream of TRCSM genes, including PIc $\beta 2$, gustducin, Ip3r3, Trpm5, and Ggamma13 in mouse, human, and rat. Ninety-four transcription factors were identified as putative transcription regulators. (B) Summary of the putative HES1 binding sites in the $5 \mathrm{~kb}$ upstream sequence of each TRCSM. The putative binding sites on the mouse, human, and rat sequences are indicated with differently colored arrows (mouse, red; human, blue; and rat, green) on the horizontal lines, which represent the $5 \mathrm{~kb}$ upstream sequences of the TRCSMs.

doi:10.1371/journal.pgen.1000443.g002

Therefore, it is important to observe HES1 colocalization with markers for other differentiated cell types within taste buds $[12,24]$. Similar results in the case of IP3R3 (Figure 4) were obtained with the blood type $\mathrm{H}$ antigen and SNAP25, which represent type I and type III cells, respectively, within taste buds (Figure 4) [12,24]. Our results raise the possibility that HES1 is commonly expressed in precursor cells involved in the cell type differentiation pathway within GVP, and that Hes 1 activity is required in the precursor or stem cell population in taste system development.

\section{Loss of Hes 1 Activity Leads to Overexpression of TRCSMs in Developing CVP}

To clarify the potential role of Hes 1 during development of the taste recognition system in vivo, we performed analyses of taste cell differentiation in mouse Hes 1 mutants. Because Hes $1^{-1-}$ mice die at the newborn stage, observations of entire CVP by serial section were conducted around the time of birth. In wild-type littermates,

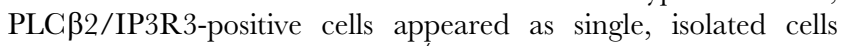
(Figure 5A). However, in Hes $1^{-1-}$ embryos, the PLCß2/IP3R3positive cells were relatively small in shape, increased in number, and in contact with one another, forming cell clusters within the CVP of E18 embryos (Figure 5A). The total number of PLC $32-$ and/or IP3R3-positive taste cells in the entire CVP was more than
A
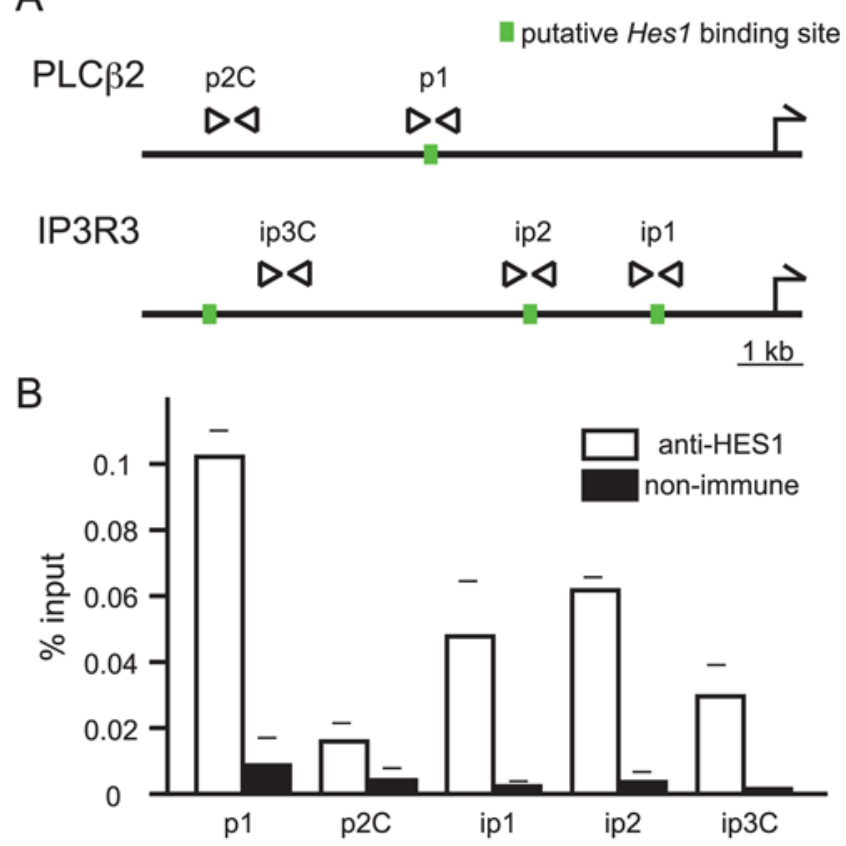

Figure 3. Binding of HES1 to TRCSM promoter sequences. (A) Position of HES1 binding sites within the promoter regions of $P / c \beta 2$ and Ip3r3 are indicated by green squares. Arrowheads indicate primer pairs used for ChIP assays. Primer pairs p1, ip1, and ip2 amplified the DNA fragment that included HES1 binding sites, while fragments amplified by $\mathrm{p} 2 \mathrm{C}$ and ip3C primer sets did not contain the HES1 binding sequence. (B) ChIP results using P19 embryonal carcinoma cells as chromatin substrate. HES1 antibody efficiently precipitated sequences containing $P / c \beta 2$ and $I p 3 r 3$ promoter HES1 binding sites.

doi:10.1371/journal.pgen.1000443.g003

5-fold greater in $\mathrm{Hes}^{-/-}$embryos than in their wild-type littermates at E18 and P0 (Figure 5).

Previous lineage tracing studies have indicated that taste cells are derived from as-yet unidentified stem cells that reside outside of taste buds, and that immature but postmitotic progenitors derived from these stem cells enter taste buds before the last division and final round of differentiation step [8-11]. Thus, the HES1 that we observed in cells within the taste buds (Figure 4) suggests that it may play a role in repressing TRC.SMs in these progenitor cells (Figure 6). These observations support our hypothesis that Hes 1 functions as a repressor of TRCSMs in taste cell precursor cells.

\section{Discussion}

Despite its importance, research regarding the molecular mechanisms of the development of the taste system has lagged behind that of the other special sense organs [3-5]. In our investigation of the molecular regulation of taste cell differentiation, we isolated key regulators of taste cell differentiation in early development by combining computational and experimental biology.

Sharing gene expression regulatory components is an efficient way of regulating molecules within the same signal transduction pathway. TRCSMs are indeed expressed in the same population of cells, at least during the course of early taste system development. We performed in silico analysis of stretches of sequence up to $5 \mathrm{~kb}$ upstream of TCRSMs, some of which had been shown previously to drive TCRSM expression in taste cells in transgenic mice $[25,26]$. Using computational analysis to determine which transcription factor binding sites were commonly found in the promoters of genes involved in the same regulatory 

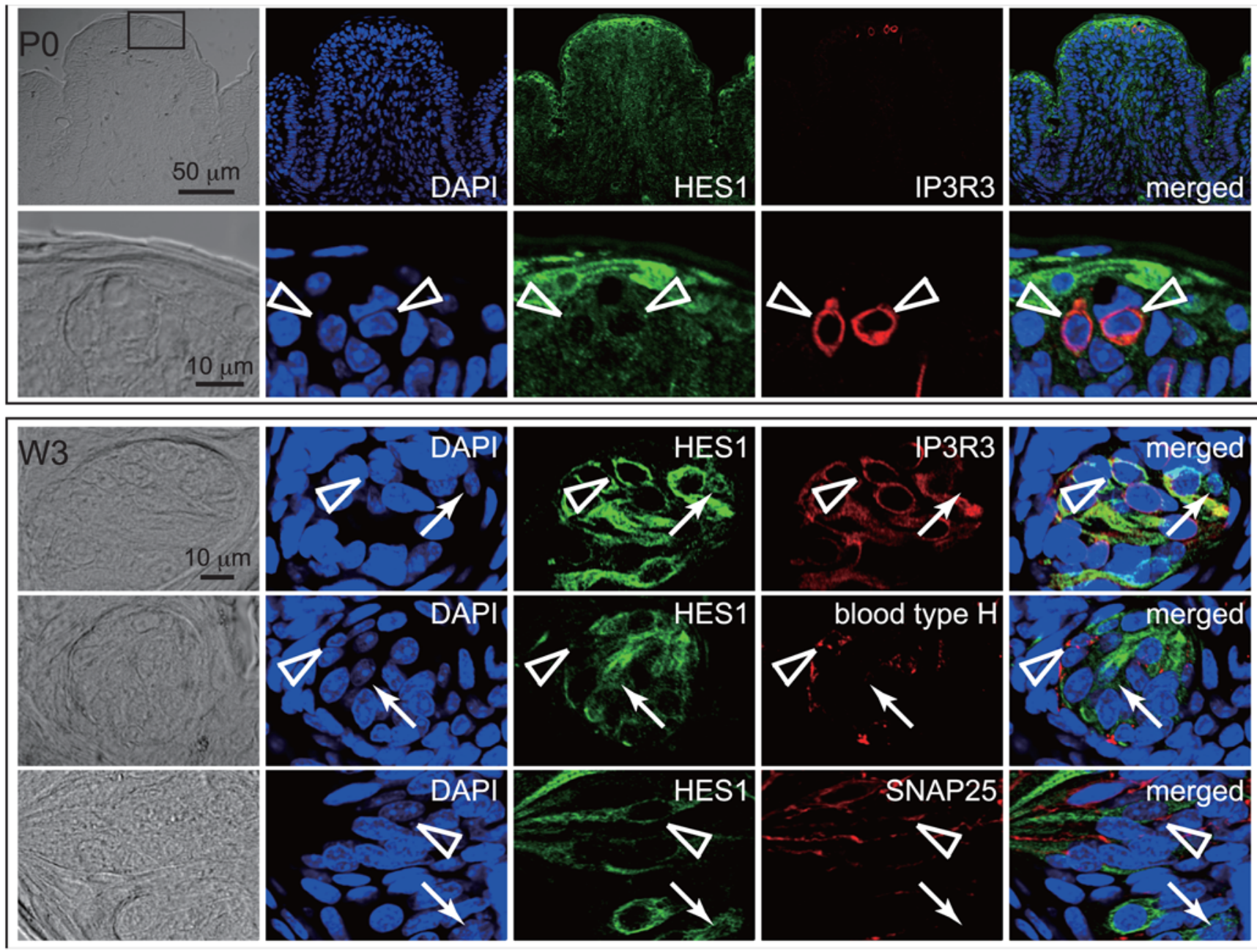

Figure 4. Immunohistochemical analysis of HES1 and IP3R3 in developing CVP at PO and W3. HES1 immunoreactivity exhibited rather uniform distribution in CVP from P0 animals, whereas a few nuclei showed reduction of HES1 immunoreactivity. The cells with reduced nuclear HES1 immunoreactivity exhibited IP3R3 expression at P0. In W3 animals, most of the cells in the taste buds displayed cytoplasmic localization of HES1, suggesting that it was nonfunctional as a transcription regulator. The very few cells retaining HES1 in the nucleus are indicated by white arrows; these cells did not express IP3R3 or SNAP25. However, some of the cells with reduced HES1 reactivity in the nucleus expressed IP3R3, SNAP25, or blood type $\mathrm{H}$ antigen (arrowheads).

doi:10.1371/journal.pgen.1000443.g004

network, we identified a number of putative transcription regulators. Similar procedures could be applied to analyses of other systems.

It has been proposed that the development of taste buds is regulated by epithelial-mesenchymal interactions involving several different signaling pathways, such as Notch, Shh, Wnt, and BMP [23,27-32]. Recent analyses of $\beta$-catenin and Sox 2 suggest that they are involved in taste cell development, although the steps involved in differentiation have yet to be clarified [27-29]. The expression patterns of Notch signaling pathway-related genes indicate that the Notch signaling cascade may have a role during morphological differentiation of CVP [23]. Here, we report that the number of TRCSM-positive cells is more than 5-fold greater in Hes $1^{-/-}$ embryos than in their wild-type littermates at stages E18 and P0 (Figure 5). Although we believe that the increase in TRCSM-positive cells observed in Hes $1^{-/-}$mutants is due to premature expression of these marker proteins in the taste cell lineage, we cannot not exclude other possibilities, such as an increase in the total number of cells in GVP, or ectopic expression in cell types other than taste cells, in which expression of TRCSMs is normally repressed by HES1.
Previous studies have proposed that a precursor population in the developing central nervous and hematopoietic systems expresses Hes 1 to maintain its undifferentiated state, and that downregulation of Hes 1 leads to differentiation [33-37]. Hes 1 may have a similar function in the taste cell lineage, and a reduction in nuclear HES 1 would thus trigger taste cell differentiation in GVP epithelium. In addition, we observed a reduction in nuclear HES 1 in blood type $\mathrm{H}$ antigen- and SNAP25-positive cells (corresponding to type I and type III taste bud cells, respectively) in older animals (Figure 4). These observations support the possibility that Hes 1 is indeed a common regulator of taste bud cell differentiation.

Our computational analysis yielded several transcription factors that may be involved in the TRCSM regulatory network (Table S2). Our investigation of HES1, one of the candidate transcription factors, provides support for the utility of the computational approach. Our list of TRCSM regulators will be a valuable resource for future studies of taste development, leading to a better understanding of the process of taste cell differentiation. Further, it may be useful for designing therapies for taste disorders, such as loss of taste. 
A
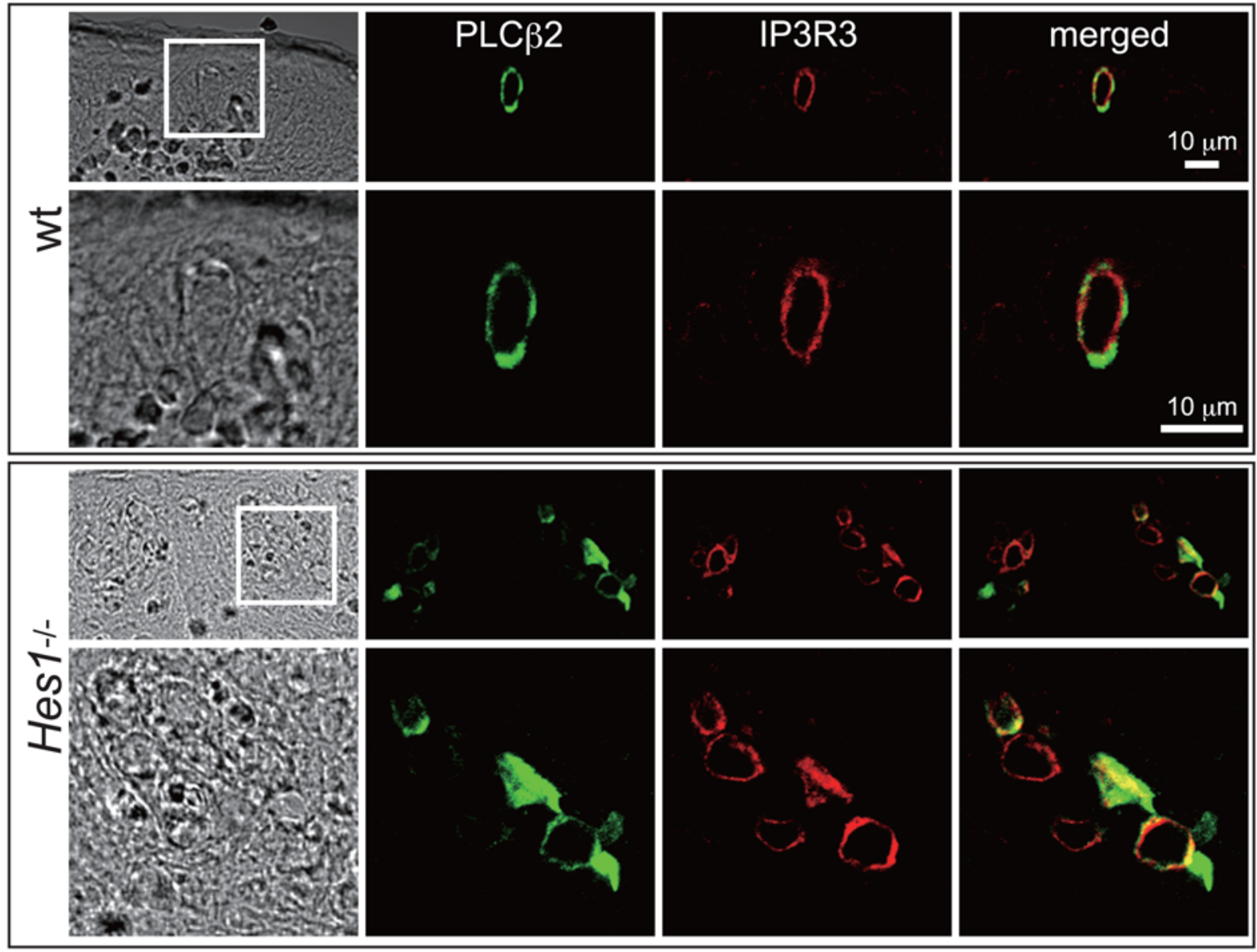

B

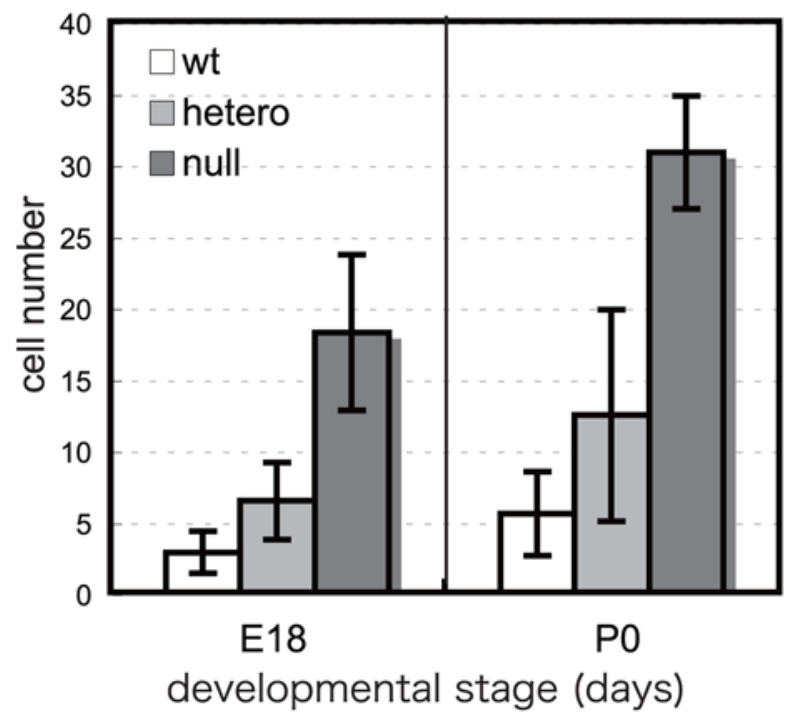

C

\begin{tabular}{c|ccc} 
stage & wt-Hes1+/+ & Hes1+/- & Hes1-/- \\
\hline E18 & $2.8(1.5)$ & $6.4(2.7)$ & $18.2(5.5)$ \\
P0 & $5.5(2.9)$ & $12.4(7.4)$ & $30.8(4.0)$
\end{tabular}

PLC $\beta 2 /$ IP3R3 positive cells on CVP of wt or Hes1 mutants mean (SD)

number of specimen for each column is more than five

Figure 5. Gene dosage effect of Hes1 on taste cell differentiation. (A) CVP from E18 embryos of wild-type and Hes $1^{-/-}$mutant littermates were stained with antibodies against PLC $\beta 2$ and IP3R3. The developing taste buds from the oral epithelium of Hes $1^{-1-}$ mutants (lower panels) exhibited many more PLC $\beta 2$ (green) and/or IP3R3 (red) immunoreactive cells than did their wild-type littermates (upper panels), which displayed very few PLC 32 and IP3R3 immunoreactive cells at this stage. Scale bar, $10 \mu \mathrm{m}$. (B) PLC 32 and IP3R3 immunoreactive cells in Hes 1 mutant CVP at E18 and P0. Serial sections of entire CVP from wild-type, Hes $1^{+/-}$, and Hes $1^{-/-}$littermates were immunostained with the PLC $\beta 2$ and IP3R3 antibodies, and immunoreactive cells were counted. The results represent the mean of more than five specimens. (C) The table shows the average and standard deviation (S.D.) of PLC 32 and IP3R3 immunoreactive cells at E18 and PO obtained from sections of entire CVP from wild-type, Hes $1^{+/-}$, and Hes $1^{-/}$ littermates. The table is graphically displayed in Figure 5B. More than five specimens of each genotype and stage were used for counting the cells. doi:10.1371/journal.pgen.1000443.g005 


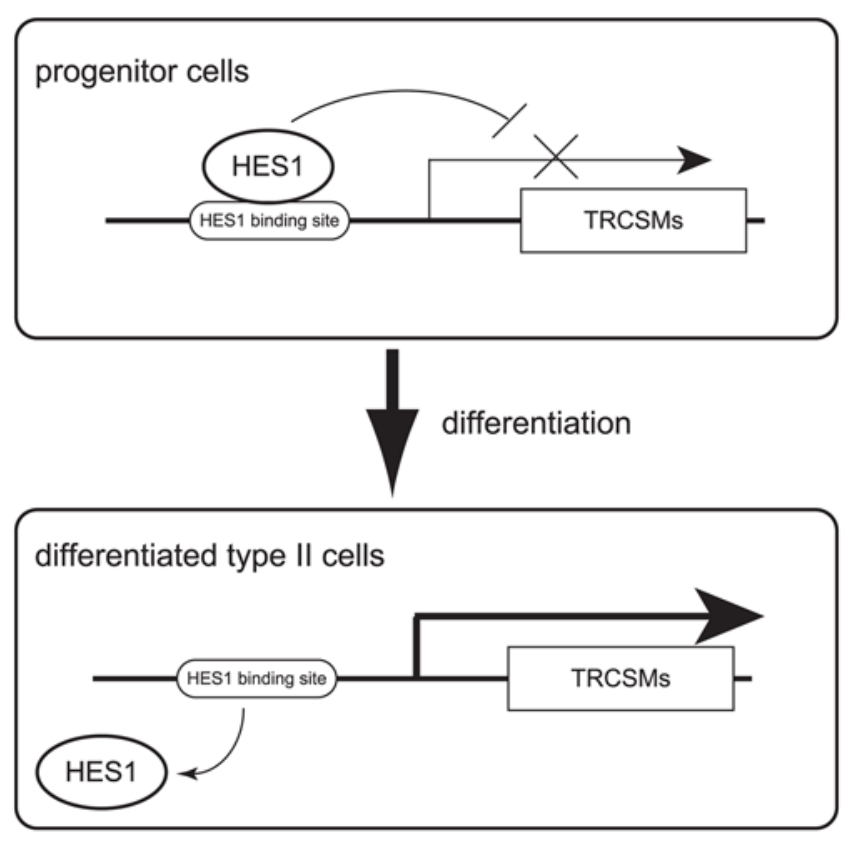

Figure 6. Schematic illustration of the transcriptional regulation of TRCSMs during differentiation of taste cells. HES1 activity is required to maintain the undifferentiated state and to repress the transcription of TRCSMs in developing immature taste cells. Loss of Hes 1 is accompanied by differentiation of taste cells expressing TRCSMs such as PLC $\beta 2$ and IP3R3.

doi:10.1371/journal.pgen.1000443.g006

\section{Materials and Methods}

\section{Animals and Tissues}

Hes1 mutant animals were kindly provided by Ryoichiro Kageyama [37]. Developing GVP were fixed with neutralized $10 \%$ formaldehyde and embedded in paraffin. The histological protocols were described previously $[38,39]$. The sections were $7.5 \mu \mathrm{m}$ thick. Serial sections were prepared from the tongue, including entire CVP. A series of serial sections was subjected to immunohistochemistry or in situ hybridization with each combination of antibodies or probes (PLC $\beta 2$ and IP3R3, gustducin and IP3R3, Plc $\beta 2$ and Ggamma13, and Plc $\beta 2$ and Trpm5). For each combination of antibodies or probes, more than five serial section series were used for staining. Overall, tongues from more than 80 animals (four combinations of markers at stages E16, E17, P0, and P5) were analyzed to observe colocalization of TRCSMs (Figure 1).

\section{In Silico Analyses of Promoter Sequences}

The mouse, rat, and human sequences $5 \mathrm{~kb}$ upstream of the TRCSMs that we investigated were retrieved from the Ensembl v46 (Aug 2007) database: Plc 2 2(ENSMUST00000077829), Trpm5 (ENSMUST0000009390), gustducin (Gnat3) (ENSMUST000000030561), Ip3r3 (ENSMUST00000049308), and Ggamma13 (Gng13) (ENSMUST00000026836). We utilized vertebrate-specific profiles of transcription factor binding sites (TFBSs) in the TRANSFAC Professional database 11.3 (10 September 2007). We searched for putative TFBSs in the promoter sequences of five TRCSMs in mouse, rat, and human using the MATCH program (version 10.4) [22], with the option of minimizing the number of the error rates of false positives and false negatives. Among the putative TFBSs that we discovered in these cross-species searches, we identified putative TFBSs that were conserved among all three species. We also performed a database search for genes expressed during stages E16-E18 in mouse undifferentiated oral epithelium in the Mouse Genome Informatics page of Jackson laboratory (as salivary gland precursor cells and oral epithelium at Theiler's Stage (TS) 24-TS26) (http://www.informatics.jax.org/) (Figure S3).

\section{ChIP}

ChIP experiments were performed in accordance with previous reports $[40,41]$ and a technical protocol established by the Farnham laboratory (http://genomecenter.ucdavis.edu/farnham/ farnham/protocols/tissues.html). We used stage P19 embryonal carcinoma cells as a substrate for ChIP assays, and an antibody against HES1 (Chemicon, AB5702: antibody raised against a synthetic peptide).

The following primers were used: pl pair, 5'-TGTTAGAACGCTGGAGTTCAAG-3' and 5'-ATCAGGCTCAGCTTTCGCATG-3'; p2C pair, 5'-AAAGTCTCTCGGACACG-

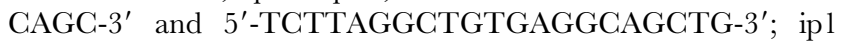
pair, 5'-GAGGAGAATGAGATCGGCATC-3' and 5'ACTGGGTAGCTGCTGCTACAG-3'; ip2 pair, 5'-CTCATTGACACGTGGGAGGAG-3' and 5'-GGAATCTACATCGCTCAGTGG-3'; and ip3C pair, 5'-GTTGGGTCCAGAGTCAGAGAC-3' and 5'-CTCACCTTCTAGGATCTCAGG-3' .

\section{Immunostaining}

We used antibodies to PLCß2 (Santa Cruz, SC206: antibody raised against amino acids 1170-1181 of PLC $\beta 2$ of human origin), gustducin (Santa Cruz, SC395: antibody raised against amino acids 93-112 of gustducin of rat origin), IP3R3 (BD Transduction Laboratories, 610312: antibody raised against amino acids 22-230 of IP3R3 of human origin), SNAP25 (Abcam, ab24737: antibody raised against full length protein-the critical epitope lies aminoterminal of the C-terminal peptide), and human blood type $\mathrm{H}$ antigen $(\mathrm{AbH})(\mathrm{Abcam}, \mathrm{ab} 3355$ : antibody raised against human colon cancer cell line SW-403). An antibody against HES1 was raised in this study using a polypeptide corresponding to amino acids $24-41$ of HES1 (TPDKPKTASEHRKSSKPI) to immunize a rabbit and produce anti-HES1 antibody (Figure S5). Antiserum was purified by the same polypeptide. We verified the specificity of this antibody by western blotting and immunohistochemistry on the spinal cords of wild-type and Hes ${ }^{-1-}$ embryos (Figure S5). Our anti-HES1 antibody recognized nuclear localized HES1 in neurons from the embryonic spinal cord in wild-type animals (Figure S5).

All sections were treated with HistoVT One solution (Nakalai Tesque, 06380-05) for antigen retrieval. Images were captured with LSM51 confocal microscopy (Zeiss), and their optical thicknesses are $1 \mu \mathrm{m}[42]$.

\section{Estimation of Immunoreactive Cells}

All PLCB2-, gustducin-, and IP3R3-positive cells were counted in $7.5 \mu \mathrm{m}$ serial immunohistological sections from whole CVP. Immunoreactive cells were counted only when nuclear staining with DAPI was clearly observed in the same cell. Immunofluorescence that appeared at a similar position in two successive sections was counted as one positive cell. All immunoreactive cells were observed with an LSM51 confocal microscope (Zeiss) and the optical thicknesses of images are $1 \mu \mathrm{m}$ [42].

\section{Supporting Information}

Figure S1 Expression of PLC $\beta 2$ and CK8 in CVP epithelium at E17. Double color immunohistochemistry against CK8 (green) and PLC $\beta 2$ (red) in CVP at E17 revealed appearance of PLC $\beta 2$ positive cells within CK8 positive cell population. 
Found at: doi:10.1371/journal.pgen.1000443.s001 (0.1 MB PDF)

Figure S2 Colocalization of TRCSMs in developing CVP. Plc $\beta 2 /$ Ggamma 13 or Trpm 5 expression in developing taste buds in the CVP from stages E17 to P5 was examined by double-color fluorescent in situ hybridization. Plc $\beta 2$ (green) and Ggamma13 or Trpm5 (red) signals always colocalized in the same cells, at least until P5. Scale bar, $10 \mu \mathrm{m}$.

Found at: doi:10.1371/journal.pgen.1000443.s002 (0.3 MB PDF)

Figure S3 Experimental strategy for in silico analysis to identify putative common regulatory factors of TRCSMs. The flowchart indicates an experimental strategy of using in silico analysis to identify the putative common regulatory factors of TRCSMs.

Found at: doi:10.1371/journal.pgen.1000443.s003 (0.01 MB PDF)

Figure S4 In situ hybridization of Hes 1 in CVP epithelium. Hes 1 expression was stronger in the deep trench epithelial cells during early development of CVP (E17 and P0). In adults (10 wk after birth, W10), cells in taste buds strongly expressed Hes1. The rectangle in the third image indicates the field shown in the rightmost image. Scale bars for the left three images, $100 \mu \mathrm{m}$. Scale bar for the right-most image, $50 \mu \mathrm{m}$.

Found at: doi:10.1371/journal.pgen.1000443.s004 (0.05 MB PDF)

Figure S5 Evaluation of the anti-HES1 antibody. We evaluated our anti-HES1 antibody by Western blotting and immunohistochemistry. Western blotting was carried out against a lysate of $\cos 7$ cells with a pCMV expression vector DNA without insert (mock, lane 1) and a DNA construct expressing a FLAG-HES1 fusion protein under the control of the CMV promoter (lane 2). The filter on the left was incubated with anti-HES1 antibody, and the filter on the right was incubated with anti-FLAG antibody (Sigma). The same band at about $30 \mathrm{kDa}$ reacted against the antibody, suggesting that the band corresponded to the FLAG-HES1 fusion protein. Immunohistochemical tests were also carried out with $H e s 1^{-/-}$mutant and wild-type siblings. The immunohistochem-

\section{References}

1. Spielman AI (1998) Chemosensory function and dysfunction. Crit Rev Oral Biol Med 9: 267-291

2. Chandrashekar J, Hoon MA, Ryba NJ, Zuker CS (2006) The receptors and cells for mammalian taste. Nature 444: 288-294.

3. Beites GL, Kawauchi S, Crocker CE, Calof AL (2005) Identification and molecular regulation of stem cells in the olfactory epithelium. Exp Cell Res 306: 309-316.

4. Kelley MW (2006) Hair cell development: commitment through differentiation. Brain Res 1091: 172-185.

5. Limb GA, Daniels JT, Cambrey AD, Secker GA, Shortt AJ, et al. (2006) Current prospect for adult stem cell-based therapies in ocular repair and regeneration. Curr Eye Res 31: 381-390.

6. Zhang Y, Hoon MA, Chandrashekar J, Mueller KL, Cook B, et al. (2003) Coding of sweet, bitter, and umami tastes: different receptor cells sharing similar signaling pathways. Cell 112: 293-301.

7. Mueller KL, Hoon MA, Erlenbach I, Chandrashekar J, Zuker CS, et al. (2005) The receptors and coding logic for bitter taste. Nature 434: 225-229.

8. Delay RJ, Kinnamon JC, Roper SD (1986) Ultrastructure of mouse vallate taste buds: ii. Cell types and cell lineage. J Comp Neurol 253: 242-252.

9. Hirota M, Ito K, Okudela K, Kawabe R, Hayashi H, et al. (2001) Expression of cyclin-dependent kinase infibitors in taste buds of mouse and hamster. Tissue Cell 33: 25-32.

10. Finger TE (2005) Cell types and lineages in taste buds. Chem Senses 30(suppl 1): i54-i55.

11. Miura H, Kusakabe Y, Harada S (2006) Cell lineage and differentiation in taste buds. Arch Histol Cytol 69: 209-25.

12. Defazio RA, Dvoryanchikov G, Maruyama Y, Kim JW, Pereira E, et al. (2006) Separate populations of receptor cells and presynaptic cells in mouse taste buds. J Neurosci 26: 3971-3980.

13. Farbman AI (1980) Renewal of taste bud cells in rat circumvallete papillae. Cell Tissue Kinet 13: 349-357.

14. Murray RG (1986) The mammalian taste bud type III cell: a critical analysis. J Ultrastruct Mol Struct Res 95: 175-188.

15. Stone LM, Tan SS, Tam PPL, Finger TE (2002) Analysis of cell lineage relationships in taste buds. J Neurosci 22: 4522-4529. istry of a spinal cord around floor plate from a wild-type embryo exhibited fluorescent signals in the nucleus, in the same pattern as with in situ hybridization signals. However, no obvious signals were observed from the spinal cords of Hes ${ }^{-\prime-}$ mutants. This suggests that the anti-HES1 antibody we raised exhibits HES1-specific immunoreactivity.

Found at: doi:10.1371/journal.pgen.1000443.s005 (0.09 MB PDF)

Table S1 TRCSM-positive cells in CVP at E17. We counted the cells positive for TRCSMs in early developing CVP from E17 embryos. The table indicates the number of CMVs subjected to two-color fluorescent histological analysis with each combination of markers, the total number of cells positive for each TRCSM tested, and the number of overlapping signals from a combination of two TRCSMs.

Found at: doi:10.1371/journal.pgen.1000443.s006 (0.04 MB PDF)

Table S2 Transcription factors identified by in silico analyses. Ninety-four transcription factors were identified by in silico analyses to be likely members of the TRCSM regulatory network. Found at: doi:10.1371/journal.pgen.1000443.s007 (0.03MB PDF)

\section{Acknowledgments}

We are grateful to Ryoichiro Kageyama, Shin-ichi Nishikawa, and Masatake Osawa for providing us with samples from $H e s 1^{-\prime-}$ and $H e s 1^{+\prime}$ mutant mice. We thank Keiko Abe and Yuzo Ninomiya for helpful discussions and Tomomi Shimogori for technical advice. We also thank Denis Duboule for comments on the manuscript, and members of the Kondo laboratory for sharing resources.

\section{Author Contributions}

Conceived and designed the experiments: MSO TK. Performed the experiments: MSO KK TK. Analyzed the data: MSO YK KK SO HT TK. Contributed reagents/materials/analysis tools: YK KK SO HT KE TK. Wrote the paper: MSO TK.

16. Miura H, Kato H, Kusakabe Y, Ninomiya Y, Hino A (2005) Temporal changes in NCAM immunoreactivity during taste cell differentiation and cell lineage relationships in taste nuds. Chem Senses 30: 367-375.

17. Lee MJ, Kim JY, Lee SI, Sasaki H, Lunny DP, et al. (2006) Association of Shh and Ptc with keratin localization in the initiation of the formation of circumvallate papilla and von Ebner's gland. Cell Tissue Res 325: 253-261.

18. Mbiene JP, Roberts JD (2003) Distribution of Keratin 8-containing cell clusters in mouse embryonic tongue: Evidence for a prepattern for taste bud development. J Comp Neurol 457: 111-122.

19. Miura H, Nakayama A, Shindo Y, Kusakabe Y, Tomonari H, et al. (2007) Expression of gustducin overlaps with that of type III IP3 receptor in taste buds of the rat soft palate. Chem Senses 32: 689-96.

20. Huang L, Shanker YG, Dubauskaite J, Zheng JZ, Yan W, et al. (1999) Ggamma13 colocalizes with gustducin in taste receptor cells and mediates IP3 responses to bitter denatonium. Nat Neurosci 2: 1055-1062.

21. Miyoshi MA, Abe K, Emori Y (2001) IP(3) receptor type 3 and PLCbeta2 are co-expressed with taste receptors $\mathrm{T} 1 \mathrm{R}$ and $\mathrm{T} 2 \mathrm{R}$ in rat taste bud cells. Chem Senses 26: 259-265.

22. Kel AE, Gössling E, Reuter I, Cheremushkin E, Kel-Margoulis OV, et al. (2003) MATCH: A tool for searching transcription factor binding sites in DNA sequences. Nucleic Acids Res 31: 3576-3579.

23. Seta Y, Seta C, Barlow LA (2003) Notch-associated gene expression in embryonic and adult taste papillae and taste buds suggests a role in taste cell lineage decisions. J Comp Neurol 464: 49-61.

24. Pumplin DW, Getschman E, Boughter Jr JD, Chengsi Y, Smith DV (1999) Differential expression of carbohydrate blood-group antigens on rat taste-bud cells: relation to functional marker a-gustducin. J Comp Neurol 415: 230239.

25. Wong GT, Ruiz-Avila L, Margolskee RF (1999) Directing gene expression to gustducin-positive taste receptor cells. J Neurosci 19: 5802-5809.

26. Kim JW, Roberts G, Maruyama Y, Berg S, Roper S, et al. (2006) Faithful expression of GFP from the PLCbeta2 promoter in a functional class of taste receptor cells. Chem Senses 31: 213-219.

27. Okubo T, Pevny LH, Hogan BL (2006) Sox2 is required for development of taste bud sensory cells. Genes Dev 20: 2654-2659. 
28. Liu F, Thirumangalathu S, Gallant NM, Yang SH, Stoick-Cooper CL, et al. (2006) Wnt-beta-catenin signaling initiates taste papilla development. Nat Genet 39: $106-112$.

29. Iwatsuki K, Liu HX, Grónder A, Singer MA, Lane TF, et al. (2007) Wnt signaling interacts with Shh to regulate taste papilla development. Proc Nat Acad Sci USA 104: 2253-2258.

30. Bitgood MJ, McMahon AP (1995) Hedgehog and Bmp genes are coexpressed at many diverse sites of cell-cell interaction in the mouse embryo. Dev Biol 172: $126-138$.

31. Hall JM, Bell ML, Finger TE (2003) Disruption of sonic hedgehog signaling alters growth and patterning of lingual taste papillae. Dev Biol 255: 263-277.

32. Liu HX, Maccallum DK, Edwards C, Gaffield W, Mistretta CM (2004) Sonic hedgehog exerts distinct, stage-specific effects on tongue and taste papilla development. Dev Biol 276: 280-300.

33. Kageyama R, Ohtsuka T, Tomita K (2000) The bHLH gene Hes 1 regulates differentiation of multiple cell types. Mol Cell 10: 1-7.

34. Kunisato A, Chiba S, Nakagami-Yamaguchi E, Kumano K, Saito T, et al. (2003) HES-1 preserves purified hematopoietic stem cells ex vivo and accumulates side population cells in vivo. Blood 101: 1777-1783.

35. Hatakeyama J, Bessho Y, Katoh K, Ookawara S, Fujioka M, et al. (2004) Hes genes regulate size, shape and histogenesis of the nervous system by control of the timing of neural stem cell differentiation. Development 131: 5539-5550.
36. Moriyama M, Osawa M, Mak SS, Ohtsuka T, Yamamoto N, et al. (2006) Notch signaling via Hes 1 transcription factor maintains survival of melanoblasts and melanocyte stem cells. J Cell Biol 173: 333-339.

37. Ishibashi M, Ang S L, Shiota K, Nakanishi S, Kageyama R, et al. (1995) Targeted disruption of mammalian hairy and Enhancer of split homolog-1 (HES-1) leads to up-regulation of neural helix-loop-helix factors, premature neurogenesis, and severe neural tube defects. Genes Dev 9: 3136-3148.

38. Tanemura K, Ogura A, Cheong C, Gotoh H, Matsumoto K, et al. (2005) Dynamic rearrangement of telomeres during spermatogenesis in mice. Dev Biol 281: 196-207.

39. Yamada M, Tanemura K, Okada S, Iwanami A, Nakamura M, et al. (2007) Electrical stimulation modulates fate determination of differentiating embryonic stem cells. Stem Cells 25: 562-570.

40. Weinmann AS, Bartley SM, Zhang MQ, Zhang T, Farnham PJ (2001) The use of chromatin immunoprecipitation to clone novel E2F target promoters. Mol Cell Biol 21: 6820-6832.

41. Mishra RK, Yamagishi T, Vasanthi D, Ohtsuka C, Kondo T (2007) Involvement of Polycomb-group genes in establishing HoxD temporal colinearity. Genesis 45: 570-576.

42. Wang A, Ikura T, Eto K, Ota MS (2004) Dynamic interaction of p220(NPAT) and CBP/p300 promotes S-phase entry. Biochem Biophys Res Commun 325: $1509-1516$. 